\title{
Multilocus heterozygosity and inbreeding depression in an insular house sparrow metapopulation
}

\author{
HENRIK JENSEN, ERLEND MYRE BREMSET, THOR HARALD RINGSBY and BERNT-ERIK SÆTHER \\ Centre for Conservation Biology, Department of Biology, Realfagbygget, Norwegian University of Science and Technology, NO-7491 \\ Trondheim, Norway
}

\begin{abstract}
Inbreeding causes reduction of genetic variability that may have severe fitness consequences. In spite of its potentially huge impact on viability and evolutionary processes especially in small populations, quantitative demonstrations of genetic and demographic effects of inbreeding in natural populations are few. Here, we examine the relationship between individual inbreeding coefficients $(F)$ and individual standardized multilocus heterozygosity $(H)$ in an insular metapopulation of house sparrows (Passer domesticus) in northern Norway in order to evaluate whether $H$ is a good predictor for $F$. We then relate variation in fitness (i.e. the probability of surviving from fledging to recruitment) to $F$ and $H$, which enables us to examine whether inbreeding depression is associated with a reduction in genetic variability. The average level of inbreeding in the house sparrow metapopulation was high, and there was large inter-individual variation in $F$. As expected, standardized multilocus heterozygosity decreased with the level of inbreeding. The probability of recruitment was significantly negatively related to $F$, and, accordingly, increased with $H$. However, $H$ explained no significant additional variation in recruitment rate than was explained by $F$. This suggests that $H$ is a good predictor for $F$ in this metapopulation, and that an increase in $F$ is likely to be associated with a general increase in the level of homozygosity on loci across the genome, which has severe fitness consequences.
\end{abstract}

Keywords: house sparrow, inbreeding, inbreeding depression, multilocus heterozygosity, pedigree, recruitment

Received 8 February 2007; revision accepted 4 June 2007

\section{Introduction}

It has been known since Darwin (1876) that reduced fitness occurs among offspring of related individuals. Consequently, animal and plant breeders try to avoid close inbreeding to ensure healthy and more productive livestock and crops. In natural populations, the probability of mating among related individuals will inevitably increase with decreasing population size, and inbreeding is now considered a threat for securing viable populations of threatened or endangered species (Frankham et al. 2002).

Inbreeding depression, i.e. that the offspring of related individuals show reduced fitness compared to the offspring from matings among unrelated individuals, is caused by

Correspondence: Henrik Jensen, Fax: ++47 73596100; E-mail: henrik.jensen@bio.ntnu.no an increase in the frequency of homozygotic loci (Wright 1977; Charlesworth \& Charlesworth 1987). Two hypotheses can explain how an increase in the frequency of homozygosity reduces fitness (Charlesworth \& Charlesworth 1987, 1999). According to the overdominance hypothesis, the heterozygote $\left(A_{1} A_{2}\right)$ has a larger fitness contribution compared to both homozygotes $\left(\mathrm{A}_{1} \mathrm{~A}_{1}\right.$ and $\left.\mathrm{A}_{2} \mathrm{~A}_{2}\right)$ and inbreeding will decrease the frequency of those superior genotypes. The partial dominance hypothesis proposes that the negative effect of increased homozygosity is caused by a fixation of recessive or partially recessive deleterious alleles in inbred lines. Some evidence suggests that many cases of inbreeding depression in natural populations are best explained by the partial dominance hypothesis (Charlesworth \& Charlesworth 1987, 1999).

Whatever genetic mechanism, a steadily increasing number of studies covering a large number of vertebrate species have shown that many fitness-related traits are 
affected by the level of inbreeding when appropriate data sets have been used (Van Noordwijk \& Scharloo 1981; Jiménez et al. 1994; Keller 1998; Crnokrak \& Roff 1999; Keller \& Waller 2002; Keller et al. 2002; Kruuk et al. 2002; Armbruster \& Reed 2005; Bensch et al. 2006; Charpentier et al. 2006; Marr et al. 2006; Smith et al. 2006). Consequently, this provides support for the notion that inbreeding is an important issue in the conservation of small and isolated populations (Soulé 1986; Keller \& Waller 2002), in captive breeding of endangered species (Ralls et al. 1988), as well as for the preservation of genetic variation (Charlesworth \& Hughes 2000; Willi et al. 2006)

Wright (1921) defined the inbreeding coefficient, $F$, to calculate the level of inbreeding for individuals in a pedigree. It is usually expressed as the probability of two homologous genes in an individual being identical by descent (Malècot 1969; Wright 1969; Crow \& Kimura 1970). Thus, individuals are considered inbred if their parents share a common ancestor.

Although quantification of the effects of inbreeding is important for understanding genetic changes and evolutionary dynamics of especially small populations, relatively few studies are available from natural populations. A major reason for this is the difficulty associated with obtaining good estimates of inbreeding in the wild. To estimate individual levels of inbreeding and its effect in wild populations, one must follow the reproduction and survival of a large proportion of the individuals in a population over several generations to construct a genetic pedigree (Balloux et al. 2004). The use of molecular genetic tools and genetic parenthood analyses is necessary in this process, because the occurrence of intraspecific brood parasitism and extra-pair copulations may result in a difference between social and genetic parenthood (Hughes 1998; Griffith et al. 2002; Jones \& Ardren 2003; Garant \& Kruuk 2005). Because there are very few existing data sets that are extensive enough to construct a reliable pedigree, scientists have tried to find alternative ways of estimating individual levels of inbreeding. The most widely used alternative has been the estimation of levels of genetic heterozygosity as a surrogate measure of inbreeding (e.g. Saccheri et al. 1998; Coltman et al. 1999; Slate et al. 2000; Acevedo-Whitehouse et al. 2003; Markert et al. 2004). The reasoning behind this is that inbreeding results in a decrease of the frequency of heterozygotes in the population (Wright 1977). However, some scepticism about the reliability of heterozygosity as an inbreeding measure has arisen during the last couple of years (Balloux et al. 2004; Pemberton 2004; Slate et al. 2004). The reason for the criticism is among other factors that an individual can be homozygous at a locus without the alleles being identical by decent. This means that outbred individuals are also homozygous at a variable number of loci (Slate et al. 2004). Furthermore, an offspring from a mating between first cousins is expected to be homozygous at only $6 \%$ of its loci through common ancestry. Hence, a large sample size, and a relatively inbred population with mating between close family members, is required to get significant statistical power to demonstrate a relationship between heterozygosity and inbreeding (Balloux et al. 2004).

This study has two major goals. First, we will examine how the level of inbreeding corresponds to the degree of heterozygosity in a natural island metapopulation of house sparrows (Passer domesticus), in which genetic parenthood was established based on mark-recapture and molecular genotyping on microsatellite loci of a large proportion ( $>70 \%$ of all individuals on all islands) of individually marked individuals (see Jensen et al. 2003, 2004). Second, we will use these data to examine whether the level of inbreeding and heterozygosity affect fledgling morphology and overall fitness, i.e. the probability of recruitment into the breeding population.

\section{Materials and methods}

\section{Study site and data collection}

The data used in this study were collected on the islands Hestmannøy, Gjærøy, Indre Kvarøy, Ytre Kvarøy, Nesøy and Aldra in an archipelago off the coast of Helgeland in northern Norway $\left(66^{\circ} \mathrm{N}, 13^{\circ} \mathrm{E}\right.$, Fig. 1$)$ during the years 1993-2004. In these parts of Norway, the breeding season lasts from early May to the middle of August (Ringsby et al. 1998). The house sparrows nest mainly on dairy farms and each pair lay from one to three clutches, each with an average of about five eggs. Both parents incubate the eggs for about 11 days, feed the nestlings for approximately 14 days until fledging, and usually continue feeding the fledglings for about 2 weeks after they leave the nest (Summers-Smith 1988). The house sparrow is a highly sedentary species with relatively low levels of natal dispersal (Altwegg et al. 2000; Tufto et al. 2005; Anderson 2006).

During the breeding seasons 1993-2002, the islands were searched for new nests, which were mainly placed inside barns on dairy farms, from every fifth to every eight day. When a nest was found it was visited about once a week until hatching. The day of hatching was recorded directly or estimated based on a subjective estimate of the age of nestlings at the first visit after hatching.

The fledglings were marked with a numbered aluminium ring and coloured plastic rings in a combination unique for each individual bird. Various measurements were taken for each fledgling at 8-13 days of age, corresponding to the period just before fledging. Tarsus length (to the nearest $0.1 \mathrm{~mm}$ ), wing length (to the nearest $1 \mathrm{~mm}$ ), and body mass (to the nearest $0.1 \mathrm{~g}$ ) was measured using slide callipers, a zero-stopped ruler and a Pesola spring 


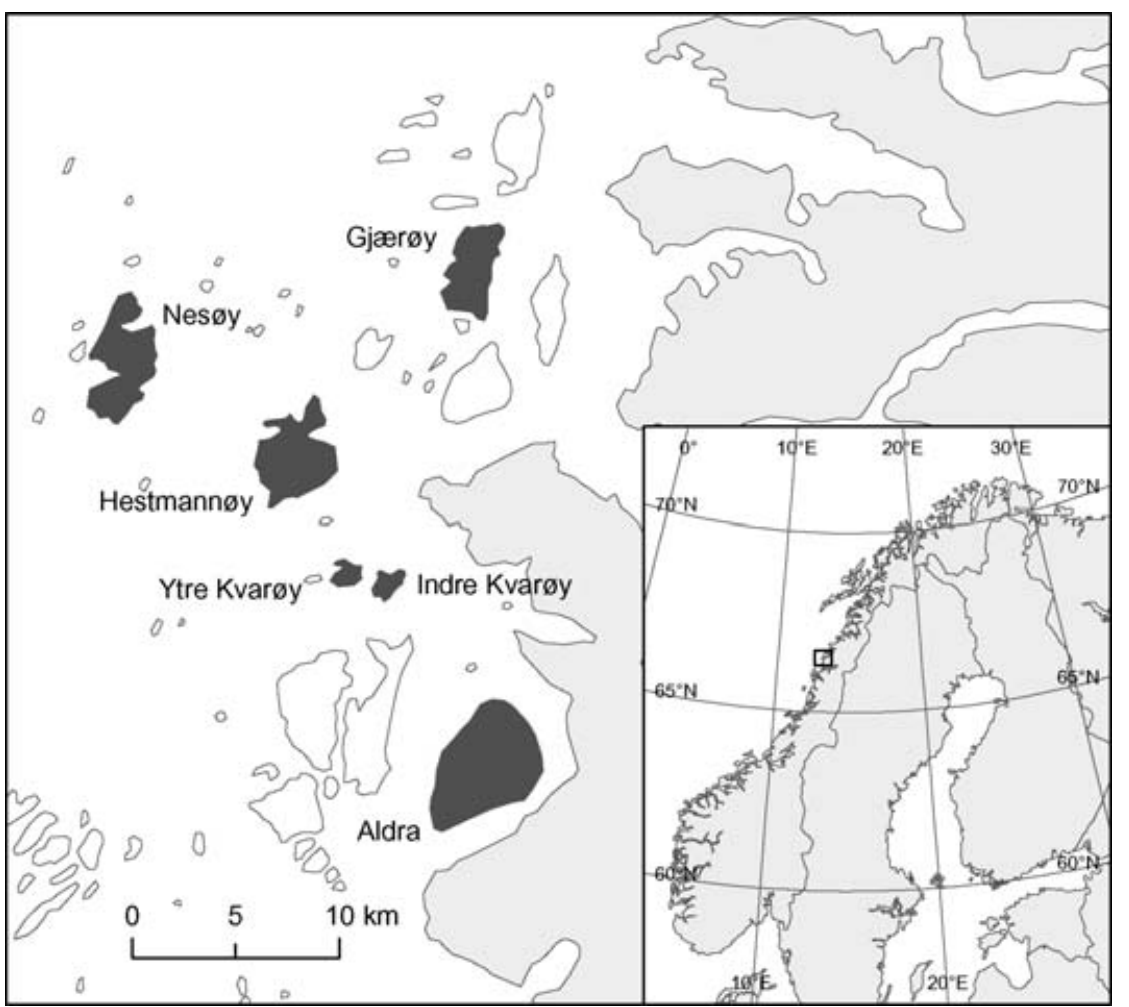

Fig. 1 Map showing the insular house sparrow metapopulation (square) off the cast of northern Norway $\left(66^{\circ} \mathrm{N}, 13^{\circ} \mathrm{E}\right)$. The six main study islands are shown in dark grey, some of the other islands in the archipelago are shown in white, and the mainland is shown in light grey. balance, respectively. Measurements were adjusted to expected size at 11 days old before statistical analyses were carried out (see Ringsby et al. 1998). Individual body condition index was estimated as the unstandardized residual from a regression of body mass on tarsus length. Finally, a small blood sample (c. $25 \mu \mathrm{L}$ ) was taken from the brachial vein of each nestling. The number of fledglings in a nest was used as a measure of clutch size in our analyses.

Fledged juveniles and adult birds were captured by mist netting and also sampled for blood. A bird was considered recruited if it was observed or recaptured a subsequent year (up until 2004). Recapture and observations was carried out on the six islands mentioned above, as well as on 12 other surrounding islands and, to some extent, on the mainland (see Ringsby et al. 1998, 1999, 2002; Sæther et al. 1999; Altwegg et al. 2000; Jensen et al. 2004; for further descriptions of the study population).

\section{Laboratory analyses}

Genetic analyses were carried out on the blood sample of each bird to determine sex, individual genotypes, and parenthood. A phenol-chloroform or a Chelex resin-based extraction method was used to extract DNA from the blood samples (Ausubel et al. 1989; Walsh et al. 1991).

The birds were genotyped by polymerase chain reaction (PCR) amplification of eight to nine highly polymorphic microsatellite loci among the loci: Esch4 (Hanotte et al.
1994), INDIGO 41 (Sefc et al. 2001), Mcy 4 (Double et al.

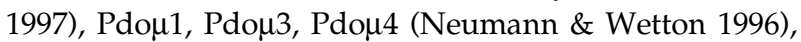

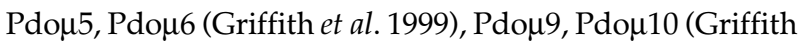
et al. 2007). A GeneAmp PCR system 9700 (Applied Biosystems) was used to carry out the reactions. The $10-\mu \mathrm{L}$ reaction solutions contained $0.5 \mathrm{U}$ of Taq DNA polymerase [Applied Biosystems (birds present 1993-1999) or Promega (birds present 2000-2002)], $20 \mathrm{~mm}\left(\mathrm{NH}_{4}\right)_{2} \mathrm{SO}_{4}$, $75 \mathrm{~mm}$ Tris- $\mathrm{HCl} \mathrm{pH} 8.8,0.15 \mathrm{mg} / \mathrm{mL}$ DNase free BSA, $10 \mathrm{~mm} \beta$-mercaptoethanol, $2.5 \mathrm{~mm} \mathrm{MgCl}_{2}, 0.2 \mu \mathrm{M}$ dNTPs (Promega), $0.7 \mu \mathrm{M}$ of each primer, and approximately $20 \mathrm{ng}$ of genomic DNA. The reverse primers were fluorescence labelled so the alleles could be resolved either on an ABI PRISM 377 Genetic Analyser (Applied Biosystems) (birds present 1993-1999) or on a 16-capillary ABI PRISM 3100 Genetic Analyser (Applied Biosystems) (birds present 2000-2002). The GENESCAN analysis software and GENOTYPER software from Applied Biosystems were used to determine individual genotypes on each of the microsatellite loci.

The sex of fledglings was determined by PCR amplification of two homologous genes (CHD1-W and CHD1-Z) located on the sex chromosomes using primers P2 and P8 (Griffiths et al. 1998). Because CHD1-W and CHD1-Z differ in size by about $50 \mathrm{bp}$ in house sparrows (Husby et al. 2006), males and females could easily be sexed as females (the heterogametic sex in birds) have two distinct bands, and males have only one band. In the sexing procedure, PCR products were separated by electrophoresis on 
$6 \%$ denaturing polyacrylamide gels and visualized by silver staining (see Husby et al. 2006 for further description of the sexing procedure).

Parenthood was determined based on individual genotypes on the microsatellite loci by using CERvus 2.0 software (Marshall et al. 1998). Because most adult birds on the study islands were marked, observations of marked birds and capture-recapture data provided information on candidate parents on each island each year. Each island was analysed separately and CERvUs also used information on the allele frequencies in each population to determine criteria that resulted in genetic parenthood which was assigned with at least $90 \%$ confidence. In the analyses, the genetic mother was determined before we determined the genetic father. In this way, a pedigree was constructed that consisted of 3536 individuals, where both genetic parents were known for $54 \%$ of the individuals (see Jensen et al. $(2003,2004)$ and Engen et al. for further description of the genotyping procedures and parenthood analyses).

\section{Inbreeding coefficient}

The individual inbreeding coefficient, $F$, of each fledgling was calculated from the pedigree using the software FSPEED PRO version 2.04 (Tenset Technologies Limited). The individual inbreeding coefficients depend on the pedigree depth (Keller 1998). Therefore, they are likely to be underestimated in many cases. All individuals with both parents known, but with an otherwise unknown family tree, will for example get an inbreeding coefficient of 0 . Consequently, to reduce this bias we only included individuals whose four grandparents were all known in our analyses.

\section{Standardized multilocus heterozygosity}

The level of heterozygosity was calculated for each individual bird (i.e. for fledglings, fledged juveniles and adults) present in the study populations from 1993 to 2002. A varying number of loci were genotyped in each individual. To make estimates of heterozygosity less biased, all individuals genotyped at fewer than seven loci were excluded from the analyses, resulting in a data set that included the genotypes of 3101 different individuals. Genotyping of individuals present during the period 19931999 was performed in a different laboratory than the genotyping of individuals present during 2000-2002. Seven of the microsatellite loci were used in both laboratories, but Esc $\mu 4$ and INDIGO41 were substituted for Pdo $\mu 9$ in the typing of birds present during 2000-2002. To avoid any bias because some of the individuals were genotyped at different loci with varying number and frequency of alleles, we estimated for each individual its standardized multilocus heterozygosity (also called standardized MLH, but hereafter denoted $H$ ) introduced by Coltman et al. (1999). Thus, $H$ was estimated based on all individuals typed at seven or more loci once or twice $(n=3299)$, as the proportion of loci an individual was genotyped for that was heterozygous, divided by the mean heterozygosity in the population at the same loci (see also Slate et al. 2004).

Out of the birds that were genotyped at seven or more loci, 198 individuals were present in both time periods, and were consequently genotyped twice. Hence, genotypes from the 2000-2002 typing were used for these birds when they were potential parents in the second time period. This ensured that the pedigrees from the two time periods were correctly linked.

At each of the seven loci common for the 1993-1999 typing and the 2000-2002 typing, from $0.5 \%$ to $17 \%$ of the individuals that were typed twice had different heterozygosity. This was due to differences between laboratories, for example different automated sequencers, different producers of chemicals and slightly different allele scoring procedures. Consequently, the heterozygosity of these individuals was significantly higher on three of the loci (Pdo $\mu 1$, Pdo $\mu 4$, and Mcy 4 4) in the 1993-1999 typing than in the 2000-2002 typing $(P<0.004)$. This was however not the case for the other four loci that were used in both periods $(P>0.5)$.

Because one microsatellite locus used in typing of birds present during 1993-1999 was replaced with two other loci in the typing of birds present during 2000-2002 (see above), only 19 individuals present during 2000-2002 were typed at exclusively the same seven loci as some $(n=101)$ of the birds present during 1993-1999. All other birds present in the last time period were typed on a different set of seven loci. Consequently, the calculation of standardized multilocus heterozygosity $H$ largely prevented any bias because of differences between laboratories (see above). Moreover, in the subsequent analyses, the $H$ from the 1993-1999 typing was used for the individuals that were typed twice because this was the period during which they hatched.

\section{Statistical analyses}

To get a more precise estimate of the individual inbreeding coefficient $F$, we excluded from the analyses all individuals with one or more unknown grandparents. Hence, we avoided including individuals that had an $F=0$ exclusively because they lacked the pedigree depth allowing for an $F>0$. Furthermore, we excluded all individuals that were genotyped on less than seven microsatellite loci. Finally, because one of our major goals was to examine the effect of inbreeding on the morphology and survival of fledglings, we only included fledglings in our analyses. The sample size on one of the islands, Ytre Kvarøy, then was only three 
fledglings. Hence, this island was excluded from the analyses. Because of these selection criteria, the data set was reduced from more than 3000 individuals to only 169 fledglings.

To examine whether variation in individual inbreeding coefficients and level of heterozygosity explained variation in morphological traits of fledglings, we applied generalized linear mixed models (GLMM) with a Gaussian error structure. These models were fitted using the lmer function and maximum-likelihood (ML) estimation procedure implemented in the lme4 and matrix libraries in $\mathrm{R}(\mathrm{R}$ Development Core Team 2004). The level of significance of each explanatory variable was determined using likelihoodratio tests (see below). Parameter estimates were obtained from the model when it was fitted using a restricted maximum-likelihood (REML) estimation procedure ( $R$ Development Core Team 2004). Furthermore, Markov Chain Monte Carlo sampling implemented in the lme4 library in $\mathrm{R}$ was used to construct confidence intervals of each estimated parameter based on 1000 samples generated from the posterior distribution of parameters in the model when it was fitted using REML (R Development Core Team 2004).

To examine whether inbreeding and heterozygosity affected the probability of recruitment of fledglings, GLMMs with a binomial error structure were applied. These models were fitted using the lmer function and the Laplace approximation implemented in the lme4 libraries in $\mathrm{R}(\mathrm{R}$ Development Core Team 2004).

In the GLMMs either a morphological trait or the recruitment probability ( 1 if recruited; 0 if not recruited) of fledglings was the response variable. $F$ or $H$ was included as an explanatory covariate when effects of individual level of inbreeding and heterozygosity were examined, respectively. In addition, any spatial or sexual differences in average morphological size or recruitment rate were accounted for by including island of hatching and fledgling sex as fixed factors in the models, respectively. Hatch day was included as covariate because this parameter has previously been shown to affect growth and survival of fledglings
(Ringsby et al. 1998; Ringsby et al. 1999; Sæther et al. 1999; Ringsby et al. 2002; Husby et al. 2006). In models where effects on morphology were examined, we also added clutch size as a covariate because this may influence the growth of offspring (Ringsby et al. 2002; Husby et al. 2006).

To test whether the effect of inbreeding on fledgling morphology or recruitment rate differed between males and females, we included the interaction term between sex and either $F$ or $H$. Finally, year of hatching and maternal identity were included as random factors to account for any effects of common environment and maternal effects, respectively. In order to identify the most important terms, we applied a stepwise-exclusion method based on likelihood-ratio tests (LRT). Accordingly, based on the global model, we successively removed the least significant terms until only explanatory variables with $P<0.1$ remained in the model. However, the main effects of $F$ or $H$, as well as the random factors were always retained in the models. The levels of significance of $\boldsymbol{F}$ and $\boldsymbol{H}$ were examined in the final model.

In addition to estimating the general effect of inbreeding $(F)$ on recruitment rate, we also wanted to examine whether the strength of any such effect differed between islands (i.e. whether the slopes differed). One way this could be determined was to estimate the level of significance of an interaction term between island of hatching and $F$, added to the final model. The full data set did not allow testing such an interaction, probably because of limited variation in $F$ among recruiting fledglings on the island Indre Kvarøy (see Table 1). However, when only fledglings on the other four islands were included in the analyses ( $n=156)$ the interaction could be tested.

The relationship between $H$ and $F$ was analysed using a GLMM with Gaussian error structure in which $H$ was the response variable and $F$ the only explanatory variable. In addition, we accounted for any interdependence between fledglings of the same mother by including maternal identity as a random factor.

Table 1 Population sizes, levels of standardized multilocus heterozygosity $(H)$, levels of inbreeding $(F)$, and recruitment rate in five island populations of house sparrows in northern Norway. Estimates are based on fledglings $(n)$ for which all four grandparents were genetically determined. Means are given \pm one standard deviation. The observed variance in $F$ among the 169 fledglings was equal to 0.00839 , and the observed variance in $H$ was equal to 0.03849 among the 3299 typed individuals (calculated from equation 1 in Slate et al. 2004)

\begin{tabular}{lllllll}
\hline Island & Adult population size & $n$ & Mean $H$ & Mean $F$ & Median $F$ & Mean recruitment rate \\
\hline Nesøy & $19.2( \pm 3.9)$ & 19 & $0.899( \pm 0.151)$ & $0.057( \pm 0.069)$ & 0.016 & $0.211( \pm 0.419)$ \\
Gjerøy & $47.9( \pm 12.2)$ & 45 & $0.960( \pm 0.208)$ & $0.025( \pm 0.072)$ & 0.000 & $0.178( \pm 0.387)$ \\
Hestmannøy & $88.6( \pm 21.7)$ & 55 & $0.981( \pm 0.151)$ & $0.004( \pm 0.019)$ & 0.000 & $0.327( \pm 0.474)$ \\
Indre Kvarøy & $40.7( \pm 11.1)$ & 13 & $0.969( \pm 0.129)$ & $0.079( \pm 0.119)$ & 0.000 & $0.077( \pm 0.277)$ \\
Aldra & $16.8( \pm 12.2) \dagger$ & 37 & $0.845( \pm 0.195)$ & $0.147( \pm 0.106)$ & 0.125 & $0.351( \pm 0.484)$ \\
All islands & $204.8( \pm 37.6)$ & 169 & $0.936( \pm 0.183)$ & $0.053( \pm 0.092)$ & 0.000 & $0.260( \pm 0.440)$ \\
\hline
\end{tabular}

*Mean adult population size during the study period (1993-2002); tonly years for which house sparrows were present on the island (i.e. 1998-2002) are included in the estimate. 
To estimate the amount of variation in the response variable that was explained by a model, we calculated the model $r_{\mathrm{L}}^{2}$ (Menard 2000), which was based on a comparison of the log-likelihood of the model and the log-likelihood of a model in which only an intercept and random factors were included. In the model where we examined the relationship between $H$ and $F$, the square root of $r_{\mathrm{L}}^{2}$ was used as an estimate of the correlation $\left(r_{\mathrm{L}}\right)$ between them. The correlation was given the same sign as the slope of their relationship.

Statistical analyses were carried out in $\mathrm{R}$ version 2.2.1 ( $\mathrm{R}$ Development Core Team 2004), all tests were two-tailed, and estimates are given \pm 1 standard deviation.

\section{Results}

Among 169 house sparrow fledglings from five islands off the coast of northern Norway, 62 fledglings (36.7\%) had an inbreeding coefficient that was larger than zero (i.e. $F>0$ ). The 169 fledglings were from a total of 83 clutches produced by 49 different females. Twenty-one of the females $(42.9 \%)$ produced the 35 clutches (42.2\%) in which at least one fledgling was inbred. In inbred clutches, the mean $F$ ranged from 0.008 to 0.344 , with a mean and median of 0.129 and 0.125 , respectively.

There was relatively large inter-individual variation in the inbreeding coefficient (Fig. 2). Furthermore, the mean level of inbreeding among fledglings varied considerably between the islands in the study metapopulation, ranging from nearly zero on Hestmannøy to close to 0.15 on Aldra (Table 1). Across all islands, the mean inbreeding coefficient of fledglings was $0.053( \pm 0.092)$ (Table 1). The most inbred fledglings had an inbreeding coefficient of 0.344 $(n=4)$. However, among the inbred fledglings, the inbreeding coefficients with highest frequency were $F=0.125$

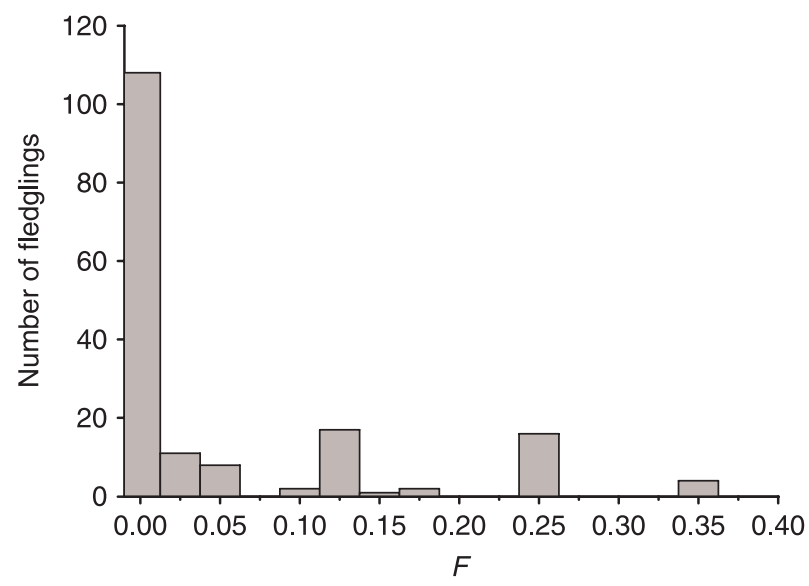

Fig. 2 Frequency distribution of individual inbreeding coefficients $(F)$ among fledglings in an insular house sparrow metapopulation off the coast of northern Norway $(n=169)$.

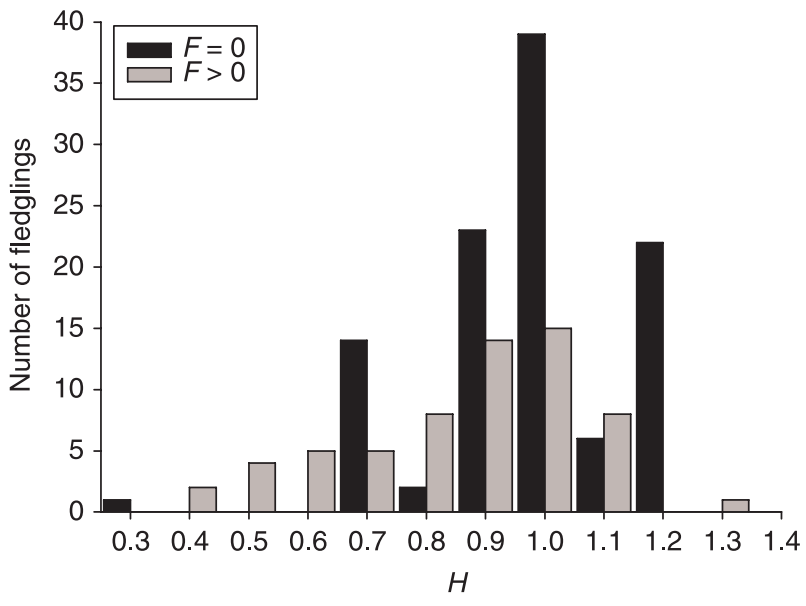

Fig. 3 Frequency distribution of standardized multilocus heterozygosity $(H)$ among fledglings in an insular house sparrow metapopulation off the coast of northern Norway $(n=169)$. The grey bars show inbred individuals $(F>0)$ and the black bars show non-inbred individuals $(F=0)$.

( $n=17)$ and $F=0.25$ ( $n=16)$, corresponding to offspring of half-sibs, and offspring of full-sibs or parent and offspring, respectively.

The standardized multilocus heterozygosity of fledglings also differed somewhat between the islands, but the variation within islands was large (Table 1). Taken across all islands, $H$ was negatively skewed (Fig. 3 ) with overall mean $H=0.936( \pm 0.183)$ (Table 1). The inbred fledglings had on average lower mean multilocus heterozygosity $(\bar{H}=0.864( \pm 0.197))$ than fledglings with $F=0(\bar{H}=0.977$ $( \pm 0.160))(t=4.067$, d.f. $=167, P<0.001$; Fig. 3$)$.

There was a highly significant negative relationship between the standardized multilocus hetereozygosity $H$ and the inbreeding coefficient $F$ of fledgling house sparrows $\left(\beta=-0.739, S E=0.172, \chi^{2}=17.622\right.$, d.f. $=1, P<0.001$, $r_{L}=-0.382$, Fig. 4). Thus, an increased level of inbreeding was associated with lower level of heterozygosity. Accordingly, we found that $F$ explained $14.6 \%$ of the variance in $H$.

\section{The effect of $\mathrm{F}$ and $\mathrm{H}$ on fledgling morphology}

There was no significant relationship between $F$ and the size of any of the morphological traits measured at fledging. This was true for models in which $F$ was entered as the only explanatory variable $(P>0.38)$, as well as after accounting for effects of hatch day, sex and/or island of hatching on variation in morphology $(P>0.38)$.

Similarly, there were no overall significant relationships between standardized multilocus heterozygosity and any of the morphological traits $(P>0.13)$. Neither was there any significant relationship between $H$ and the size of any morphological trait after accounting for effects of hatch day, sex and/or island of hatching $(P>0.11)$. 


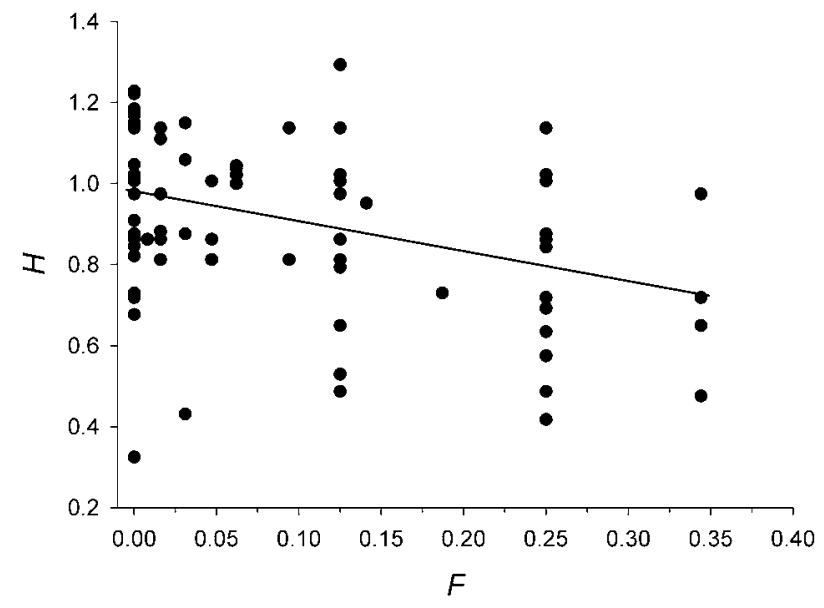

Fig. 4 The relationship between standardized multilocus heterozygosity $(H)$ and individual inbreeding coefficients $(F)$ of fledglings in an insular house sparrow metapopulation off the coast of northern Norway $(n=169)$. Any interrelationship between fledglings produced by the same mother was accounted for by including maternal identity as random factor in the model. The best fitting line was given by $H=0.981-0.739 * F ; r_{\mathrm{L}}^{2}=0.139$.

\section{The effect of $\mathrm{F}$ and $\mathrm{H}$ on the recruitment rate of fledglings}

There was no significant general relationship between the recruitment rate of fledglings and the level of inbreeding $F$ $(\beta=-3.630, \mathrm{SE}=2.532, P=0.142)$.

Previous studies from the same metapopulation have shown that recruitment rate generally differs among islands (Ringsby et al. 1998; Ringsby et al. 1999; Sæther et al. 1999; Ringsby et al. 2002), that the day of hatching may be an important determinant of recruitment probability (Ringsby et al. 1998; Ringsby et al. 2002; Husby et al. 2006), and that fledgling sex also may have an effect on recruitment (Husby et al. 2006). Accordingly, after accounting for fledgling sex, island of hatching, and hatch day (in addition to the random factors), there was a strong and significant negative relationship between the probability of recruitment and $F(\beta=-9.031, P=0.010$; Table $2 \mathrm{a}$, Fig. 5). The final model explained $10.7 \%$ of the variation in recruitment rate among fledglings on the five study islands. Our analyses also showed that there was no significant interaction between the level of inbreeding and sex $(P=0.58)$, suggesting that the general effect of inbreeding was the same in male and female fledglings. The existence of an interaction between island of hatching and $F$ could however, not be tested in the full model with all islands included because the model did not converge, probably because too few fledglings recruited on the island of Indre Kvarøy (see Table 1). When this island was excluded, our analyses showed that the interaction between $F$ and island of hatching was non-significant $\left(\chi^{2}=4.980\right.$, d.f. $=3$, $p=0.17, n=156)$. Hence, the effect of inbreeding on the probability of recruitment seemed to be similar on the
Table 2 The final models for relationships between the recruitment probability and (a) individual inbreeding coefficients $(F)$, and (b) standardized multilocus heterozygosity $(H)$ of fledgling house sparrows $(n=169)$ in an insular metapopulation off the coast of northern Norway. Table (c) shows the results when $H$ was added to the final model for $F$. Year of hatching and maternal identity were included as random factors in the models. Parameter estimates (i.e. slopes, $\beta$ ) and their standard errors (SE) are given for covariates, and the level of significance $(P)$ is given for all explanatory variables included in the final models. Below each model, the $\chi^{2}$, degrees of freedom (d.f.), level of significance, and the proportion of variance in recruitment probability explained by the final model relative to a model with only random factors $\left(r_{\mathrm{L}}^{2}\right)$ is given (see text for further details)

\begin{tabular}{|c|c|c|c|c|}
\hline & Parameter & $\beta$ & $\mathrm{SE}(\beta)$ & $P$ \\
\hline \multirow[t]{5}{*}{ (a) } & $F$ & -9.031 & 3.852 & 0.010 \\
\hline & Hatch day & 0.023 & 0.011 & 0.045 \\
\hline & Island & & & 0.076 \\
\hline & Sex & & & 0.060 \\
\hline & Model: $\chi^{2}=$ & , d.f. $=7$ & $.004, r_{\mathrm{L}}^{2}$ & \\
\hline \multirow[t]{4}{*}{ (b) } & $H$ & 2.554 & 1.263 & 0.041 \\
\hline & Hatch day & 0.026 & 0.010 & 0.011 \\
\hline & Sex & & & 0.074 \\
\hline & Model: $\chi^{2}=$ & , d.f. $=3$ & $.008, r_{\mathrm{L}}^{2}$ & \\
\hline \multirow[t]{6}{*}{ (c) } & $F$ & -7.717 & 3.920 & 0.034 \\
\hline & $H$ & 2.266 & 1.353 & 0.100 \\
\hline & Hatch day & 0.024 & 0.011 & 0.043 \\
\hline & Island & & & 0.062 \\
\hline & Sex & & & 0.037 \\
\hline & Model: $\chi^{2}=$ & , d.f. $=8$ & $.003, r_{\mathrm{L}}^{2}$ & \\
\hline
\end{tabular}

different islands. Thus, there was a significant negative effect of inbreeding on the recruitment probability of house sparrow fledglings, but this was evident only when the comparison was among fledglings experiencing the same environmental conditions.

As expected from the decrease in individual standardized multilocus heterozygosity with inbreeding (Fig. 4), the recruitment rate of fledglings increased significantly with $H(\beta=2.554, \mathrm{SE}=1.263, P=0.041)$ after accounting for the effect of hatch day and effects of sex on average probability of recruitment (Table 2b, Fig.6). The final model explained however, only $6.2 \%$ of the variation in recruitment rate of fledglings (Table 2b). There was no significant interaction between the level of heterozygosity and sex in the effect on recruitment rate $(P=0.28)$. Neither was there any significant spatial difference in average recruitment rate when $H$ was included in the model (island of hatching: $p=0.13$ ). The model that included island of hatching explained however, $9.8 \%$ of the variation in recruitment rate.

As for $F$ there was no significant general association between probability of recruitment and $H(\beta=1.732$, $\mathrm{SE}=1.130, P=0.124, n=169)$ when the effects of sex and 


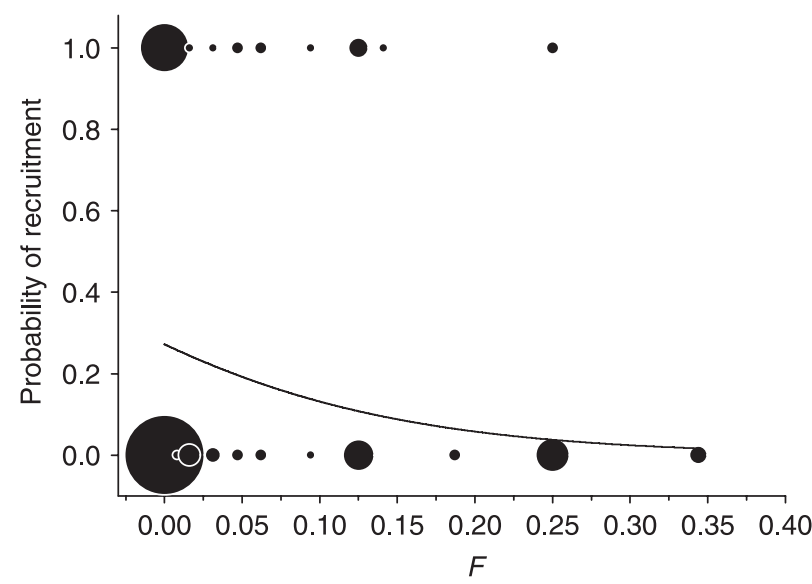

Fig. 5 The relationship between recruitment probability and individual inbreeding coefficients $(F)$ of fledglings in an insular house sparrow metapopulation off the coast of northern Norway $(n=169)$. The line shows the relationship predicted from the model presented in Table 2a for fledglings with average sex that hatched on an average island on the mean hatch day in the metapopulation. The diameter of each point is proportional to twice the square root of the number of fledglings with a given $F$ and recruitment status ( 1 if recruited; 0 if nonrecruited).

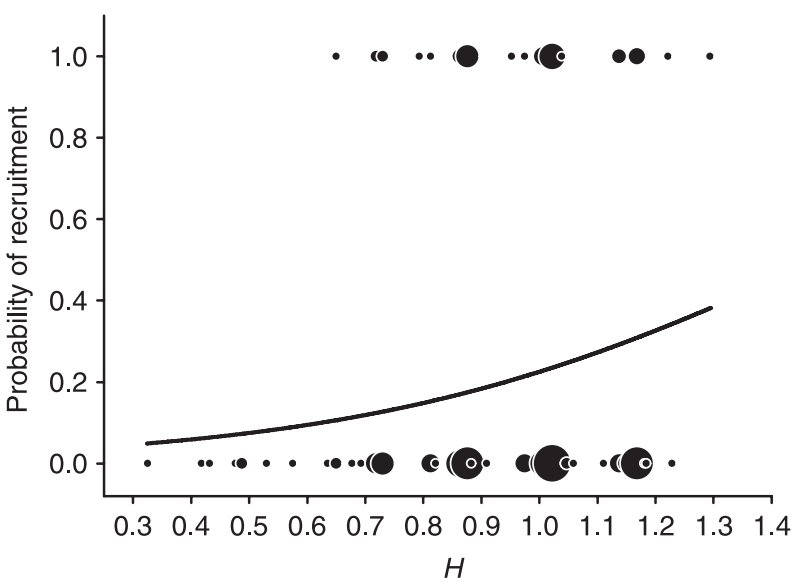

Fig. 6 The relationship between recruitment probability and standardized multilocus heterozygosity $(H)$ of fledglings in an insular house sparrow metapopulation off the coast of northern Norway $(n=169)$. The line shows the relationship predicted from the model presented in Table $2 \mathrm{~b}$ for fledglings with average sex that hatched on the mean hatch day in the metapopulation. The diameter of each point is proportional to twice the square root of the number of fledglings with a given $H$ and recruitment status (1 if recruited; 0 if nonrecruited).

hatch day on recruitment rate (see Table $2 b$ ) were not accounted for, and pooling data from all five islands.

To examine the relative contribution of $F$ and $H$ for variation in the probability of recruitment, both $F$ and $H$ were entered as explanatory variables in the final model for $F$ (see Table 2a). Hence, we could examine whether $H$ was able to explain any additional significant proportion of the residual variation in the probability of recruitment of fledglings when the variation explained by $F$ was accounted for. The analysis showed that $H$ did not explain any significant extra variation in recruitment rate beyond the variation explained by $F(P=0.100 ;$ Table 2$)$. The model which included both $F$ and $H$ explained only marginally (i.e. $1.4 \%$ ) more of the variation in the probability that fledglings recruited compared to a model with only $F$ (Table 2). Furthermore, there was no significant overall relationship between residuals from the regression of $H$ on $F$ (Fig. 2), and the probability of recruitment $(\beta=1.771, \mathrm{SE}=1.428, P=0.223)$. Lack of a significant relationship in this model remained also when the effects of sex, island of hatching, and day of hatching were accounted for $(\beta=2.196, \mathrm{SE}=1.516, P=0.096)$. Hence, our results suggest that $F$ and $H$ to a large extent explain the same variation in recruitment rate in fledgling house sparrows.

\section{Discussion}

This study shows that there was large variation in inbreeding coefficient $F$ and mulitilocus heterozogysity $H$ among individual house sparrow fledglings on five islands off the coast of northern Norway (Figs 2 and 3). Furthermore, there was a negative relationship between $H$ and $F$ (Fig. 4), suggesting that $H$ reflected the level of inbreeding in the study populations. There was also evidence of relatively strong inbreeding depression as the probability of recruitment of fledglings into the breeding population the following year decreased with increasing level of inbreeding (Table 2).

The level of inbreeding in the island metapopulation off the coast of northern Norway was among the highest ever recorded in natural populations of vertebrates. In a recent review by Slate et al. (2004), only the studies on wolves (Canis lupus, Hedrick et al. 2001; see also Ellegren 1999 and Bensch et al. 2006) and Lipizzian horses (Equus caballus, Curik et al. 2003) showed a higher mean $F$. However, the wolves descended from a captive population (Hedrick et al. 2001), and the Lipizzian horses were all bred in captivity from seven studs (Curik et al. 2003). In addition, similar levels of inbreeding as found in our house sparrow study system were found in domestic Coopworth sheep (Ovis aries, Slate et al. 2004), large ground finches (Geospiza magrinostris, Grant et al. 2001), and song sparrows (Melospiza melodia, Keller 1998; Jeffery et al. 2001).

The relatively high mean individual inbreeding coefficient demonstrated in house sparrows in this study may be caused by the biology of the house sparrow. It is a sedentary species, and it often spends its entire life in the population where it was born (Summers-Smith 1988; Anderson 2006). A previous study from the same study metapopulation has shown that less than $10 \%$ of all recruits are immigrants from neighbouring islands (Altwegg et al. 2000). Furthermore, very few house sparrows disperse further than $20 \mathrm{~km}$ away from the population where they 
hatched (Lande et al. 2003; Tufto et al. 2005). Relative to these estimates, the distance between our study islands is quite large (Fig. 1). When many individuals recruit into populations in which they were born, and these populations are relatively small, the likelihood of breeding with parents, siblings or other close relatives is high, resulting in a tendency for such populations to have a high degree of inbreeding (Caughley 1994). Some islands in the study metapopulation are thus prone to having a higher degree of inbreeding as the population size is smaller than on other islands. Accordingly, there seemed to be a negative relationship between $F$ and mean recorded population size during the study period (Table 1). Another likely reason for the high mean $F$ in this study is that one of the islands, Aldra, was colonized by only one female and three males in 1998. Not surprisingly, this population was also highly inbred (Table 1), and contributed to the high mean $F$ across islands in this study.

In addition to the fragmented population structure caused by separation of islands by sea, two of the island populations, Gjerøy and Hestmannøy, may in turn be substructured because of the relatively large distances between some of the local farms within each island. Consequently, it may be the size of these local subpopulations that govern the likelihood of mating between close relatives rather than the population size on the whole island. On the other hand, the distances between farms are considerably shorter than between islands, and although some of the farms within Gjerøy and Hestmannøy are separated by mountainous terrain, land is likely to be a weaker geographical barrier for dispersal than sea (MacArthur \& Wilson 1967). Consequently, migration between the different subpopulations (i.e. farms) may limit the amount of inbreeding. This possible additional structuring of the house sparrow populations within islands which already differ in their degree of isolation (i.e. distance from nearest neighbouring island), and in population size, may nevertheless be one possible explanation for the high variation in individual inbreeding coefficients documented in this study.

There was a negative relationship between $F$ and standardized multilocus heterozygosity in this study (Fig. 4). Inbred individuals also had a mean $H$ that was significantly lower than the mean $H$ of noninbred individuals (Fig. 3) suggesting that inbreeding reduced the level of heterozygosity within house sparrows in our study area. The mean level of absolute heterozygosity will however, vary with the type and allele frequencies of the genetic markers used. Furthermore, an individual's standardized multilocus heterozygosity $H$ is likely to differ from its unstandardized (i.e. absolute) multilocus heterozygosity because $H$ is a relative measure which depend on the average heterozyogsity of the genetic markers in the population. Finally, the mean $H$ of a study population will, per definition, be equal to one. Hence, a direct comparison of levels of heterozygosity, standardized or not, across studies becomes difficult. Nevertheless, the relationship between $H$ and $F$, as well as the relationship between these measures and fitness-related traits within studies are not affected by these problems of scale invariance.

We found that the recruitment rate of fledgling house sparrows was negatively affected by their level of inbreeding (Table 2, Fig. 5). Similar effects of inbreeding on survival of offspring have been documented in a number of other bird species (Keller et al. 1994; McRae 1996; Brown \& Brown 1998; Keller 1998; Daniels \& Walters 2000; Grant et al. 2001; Kruuk et al. 2002), as well as in other organisms (see reviews in Crnokrak \& Roff 1999; Keller \& Waller 2002). Studies have demonstrated negative effects on various other reproductive traits as well, and there seems to be a general agreement among studies that inbreeding in natural populations have negative fitness consequences (Crnokrak \& Roff 1999; Keller \& Waller 2002).

The fact that inbreeding shows an effect on recruitment and not on the morphological traits in this study fits with the findings of De Rose \& Roff (1999), who found that inbreeding in general had a stronger effect on life history traits than on morphological traits. Inbreeding also had a stronger effect on juvenile survival to recruitment than on either body size or body condition of fledglings in the collared flycatcher (Kruuk et al. 2002). Inbreeding depression is believed to be connected with effects of dominance (Charlesworth \& Charlesworth 1987, 1999). Accordingly, the fact that inbreeding generally seems to have a larger effect on survival than on morphology is believed to arise either because the amount of dominance variance in morphological traits is less than for life-history traits (Crnokrak \& Roff 1995; Roff 1998), or because the mutations affecting life-history traits are expected to have directional dominance. In contrast, mutations affecting morphological traits, which in general are only weakly correlated to fitness, are not necessarily directional dominant as the mutations lowering and increasing the trait mean are selectively equivalent (Lynch \& Walsh 1998). Moreover, in our sample of fledglings from five islands off the coast of northern Norway, none of the morphological traits measured at fledging were correlated with the probability of recruitment $(P>0.13)$. In light of the above aspects, it is thus not very surprising that inbreeding did not have a negative effect on the morphological traits in this study.

In general, $F$ and $H$ explained a similar proportion of the variation in recruitment rate (Table 2). The relatively strong effect of $F$ on fitness was found despite the shallow pedigree on which estimates of $F$ were based. If the pedigree had been deeper and more complete, an inbreeding coefficient larger than zero would probably appear in a larger proportion of the individuals, increasing the probability of detecting even weak inbreeding effects (e.g. Balloux et al. 2004). If we assume that the estimated $F$ reflects the 
true level of $F$, our analyses suggest that most of the interindividual variation in $H$ had other causes than differences among fledglings in the level of inbreeding (see Fig. 4). For example, population substructuring among and within islands may cause heterozygote deficiency due to the Wahlund effect (Hartl \& Clark 1997). In a recent study, we used 17 microsatellite loci to examine the genetic population structure of house sparrow populations along the coast of northern Norway (Moe et al., in preparation). The study showed that the pairwise $F_{\mathrm{ST}}$ between Aldra and neighbouring populations was approximately 0.062 , whereas pairwise $F_{\mathrm{ST}}$ among the neighbouring populations was about 0.017 . Hence, these results indicate that the island Aldra was genetically differentiated from neighbouring populations. As a consequence, we cannot rule out the possibility that some of the observed variation in $H$ is due to population substructure, in contrast to inbreeding. Accordingly, it is possible that population substructure contributed to the small and nonsignificant extra amount of variation in recruitment rate explained by $H$ when $F$ was accounted for (Table 2c). However, because differences between islands were accounted for when examining effects on recruitment probability, we believe that any bias due to possible population substructuring was negligible.

Three genetic mechanisms may result in a relationship between $H$ and components of fitness by relating heterozygosity at typed loci to levels of heterozygosity at loci coding for fitness traits (David et al. 1995, 1998; Savolainen \& Hedrick 1995; Lynch \& Walsh 1998; Hansson \& Westerberg 2002). The first is that the genotyped loci code for the trait in question, also called the functional or true overdominance hypothesis ('direct effect' hypothesis in Hansson \& Westerberg 2002). Alternatively, the genotyped loci are physically linked or in linkage disequilibrium with genes that code for the trait, also called associative overdominance hypothesis ('local effect' hypothesis in Hansson \& Westerberg 2002). Finally, the heterozygosity at the genotyped loci mirrors the genome-wide level of heterozygosity, that is also at unlinked loci. This last hypothesis is called the 'general effect' hypothesis (e.g. David et al. 1995; Hansson \& Westerberg 2002) and is the only hypothesis that will give a general correlation between $H$ and $F$. In contrast, the inbreeding coefficient $F$ is merely a probability of decreased heterozygosity, resulting from the probability of the same allele inherited from a common ancestor (Wright 1969; Crow \& Kimura 1970). Consequently, the fact that there are three different ways by which heterozygosity at typed loci may affect fitness could result in $H$ explaining more or an additional part of the variance in a particular fitness trait, than $F$. However, this does not necessarily make $H$ a more exact measure of inbreeding than $F$.

Based on the observed variance in $F$ among the 169 fledglings in this study, and the observed variance in $H$ among all the 3299 typed individuals (see Table 1 ), the model proposed by Slate et al. (2004) predicted that the expected value of the correlation between $F$ and $H$ should be -0.493 (i.e. using equation 4 ). The expected value is somewhat larger than the value observed in this study $(-0.381)$. The model of Slate and coworkers is, however, based on the assumptions that all loci are equally affected by inbreeding and that the typed loci are unlinked (Slate et al. 2004). Furthermore, it is assumed that all loci have the same expected heterozygosity in absence of inbreeding. The first assumption is likely to be true in most natural populations (Hartl \& Clark 1997; Frankham et al. 2002). This notion is further supported by the fact that we used microsatellite loci, which in general are assumed to be neutral (i.e. noncoding) loci located in the introns in the genome (Beebee \& Rowe 2004; but see, e.g. Kashi \& Soller 1999). Furthermore, results from a study where passerine microsatellites were mapped on the draft chicken Gallus gallus genome sequence (Dawson et al. 2006) and a linkage mapping study of great reed warbler (Acrocephalus arundinaceus) (Hansson et al. 2005) show that the loci used in this study were located on at least five different chromosomes (see also Griffith et al. 2007). This suggests that many of the typed loci used here are unlikely to be physically linked with each other. Moreover, the microsatellite loci were originally selected in order to provide high power in parenthood analyses (Jensen et al. 2003, 2004). As a consequence, they were all very variable with roughly the same level of variation. Accordingly, the observed heterozygosity in the 3299 typed individuals ranged from 0.57 to 0.95 , with a mean of 0.80 (H. Jensen, unpublished results). On the basis of the above observations, we believe that the assumptions of the model are likely to be fulfilled. The small discrepancy between the expected and observed correlations between $F$ and $H$ may be a consequence of the fact that individual inbreeding coefficients $F$ estimated from the relatively shallow pedigree are likely to be underestimated relative to true values (see above). However, the discrepancy between expected and observed correlations between $H$ and $F$ was relatively small and our results suggested that $F$ and $H$ explained largely the same variation in recruitment rate. Therefore, a genome-wide decrease in the level of heterozygosity due to inbreeding seems probable, and the 'general effect' hypothesis seems the most likely explanation for the positive relationship between $\mathrm{H}$ and fitness demonstrated here (Table 2b, Fig. 6).

Although many studies have documented significant associations between fitness traits and some measure of heterozygosity (reviewed in Hansson \& Westerberg 2002; Coltman \& Slate 2003; Slate et al. 2004), a strong concordance in expected and observed correlations between $H$ and $F$, seems to be the exception rather than the rule (Ellegren 1999; Markert et al. 2004; Slate et al. 2004; Bensch et al. 2006). Other studies have generally found that the observed correlation was considerably weaker than expected (Slate 
et al. 2004; Overall et al. 2005). In addition, results from recent studies on great reed warbler (Hansson et al. 2001, 2004), red deer (Cervus elaphus Slate \& Pemberton 2002; Slate et al. 2002) and Drosophila melanogaster (Ferreira \& Amos 2006) provide evidence that associative overdominance may be a common reason for heterozygosity-fitness correlations in animals. Out of the seven studies reported in Slate et al. (2004), only wolves and large ground finches had expected correlations between $H$ and $F$ that complied with expectations from the theoretical model. The common denominator of these two studies and our study on house sparrows is relatively small population sizes and relatively recent founder events (for one of our study populations) (Ellegren 1999; Grant et al. 2001; Hedrick et al. 2001; Bensch et al. 2006). Hence, all three studies had relatively large variances in $F$, and relatively large fitness effects of inbreeding (Table 2; Grant et al. 2001; Hedrick et al. 2001; Bensch et al. 2006), which may be a prerequisite for a strong relationship between $H$ and $F$ (Slate et al. 2004). However, especially in small populations, one cannot rule out the possibility that associative overdominance has an effect solely on the basis of the correspondence between observed and expected correlations between $H$ and $F$. This is because linkage disequilibrium is expected to be common in such populations, and may result in an association between $H$ and fitness regardless of the actual level of inbreeding (Balloux et al. 2004; Markert et al. 2004; Ferreira \& Amos 2006).

To conclude, we have shown that the inbreeding depression was relatively strong, and resulted in a reduced recruitment probability of fledglings in an insular metapopulation of house sparrows off the coast of northern Norway. There was also a significant negative relationship between $H$ and $F$. Thus, $H$ seems to be a relatively good predictor of the individual level of inbreeding in these populations. Furthermore, $H$ explained a significant part of the variation in fitness, but not any significant proportion that was not explained by $F$. This suggests that the main effect of $H$ was to provide an indication of the genome-wide level of heterozygosity in our populations. The general presence of such a relationship in natural populations may however, depend closely on the level of inbreeding in the population (e.g. Slate et al. 2004) in addition to the specific loci that are used in estimating $H$ (i.e. the degree of physical linkage with loci that affect fitness). However, one of the probable reasons for the correlation between $H$ and $F$, small population size, makes it unclear whether $H$ is likely to be a good predictor of the level of inbreeding in general (Balloux et al. 2004; Ferreira \& Amos 2006).

Our results show that thorough understanding of the effects of inbreeding in small or threatened populations is crucial for the conservation of small populations. The level of heterozygosity may affect probability of extinction regardless of the mechanism reducing heterozygosity (Frankham 1998; Saccheri et al. 1998), and populations also show different effects of inbreeding on fitness (Pray et al. 1994; Hedrick \& Kalinowski 2000). Further studies are clearly needed to determine whether associative overdominance or 'general effects' is the major reason for the effects of heterozygosity on fitness in general (see also Hansson \& Westerberg 2002), but it seems that more long-term research and construction of reliable genetic pedigrees are needed to study the fitness consequences of inbreeding in natural populations.

\section{Acknowledgements}

We would like to thank R. Altwegg, T. Berge, T. Kolaas, S. Krogstad, A. Loraas, M. Mørkved, N. M. Pedersen, E. J. Solberg, T. SvorkmoLundberg, K. Sørensen, and I. R. K. Stewart for assistance in the field, H. Ellegren, S. C. Griffith, A. Husby, L. K. Larsen and S. Skjelseth for help with the laboratory analyses, and I. Herfindal for making the map. Constructive comments from four anonymous referees and Jon Slate improved the manuscript. We also want to thank the friendly people at Helgeland, whose cooperation made this study possible. The study was supported by grants from the Norwegian Research Council (STORFORSK), Strategic University Program (SUP) in Conservation Biology the Norwegian Directorate for Nature Management, the EU-commission (METABIRD).

\section{References}

Acevedo-Whitehouse K, Gulland F, Greig D, Amos W (2003) Disease susceptibility in California sea lions. Nature, 422, 35.

Altwegg R, Ringsby TH, Sæther B-E (2000) Phenotypic correlates and consequences of dispersal in a metapopulation of house sparrows Passer domesticus. Journal of Animal Ecology, 69, 762-770.

Anderson TR (2006) Biology of the Ubiquitous House Sparrow: From Genes to Populations. Oxford University Press, New York.

Armbruster P, Reed DH (2005) Inbreeding depression in benign and stressful environments. Heredity, 95, 235-242.

Ausubel FM, Brent R, Kingston RE et al. (1989) Current Protocols in Molecular Biology. John Wiley \& Sons, New York.

Balloux F, Amos W, Coulson T (2004) Does heterozygosity estimate inbreeding in real populations? Molecular Ecology, 13, 3021-3031.

Beebee T, Rowe G (2004) An Introduction to Molecular Ecology. Oxford University Press, Oxford, UK.

Bensch S, Andrén H, Hansson B et al. (2006) Selection for heterozygosity gives hope to a wild population of inbred wolves. PLoS One, 1, e72.

Brown JL, Brown ER (1998) Are inbred offspring less fit? Survival in a natural population of Mexican jays. Behavioral Ecology, 9, 60-63.

Caughley G (1994) Directions in conservation biology. Journal of Animal Ecology, 63, 215-244.

Charlesworth D, Charlesworth B (1987) Inbreeding depression and its evolutionary consequences. Annual Review of Ecology and Systematics, 18, 237-268.

Charlesworth D, Charlesworth B (1999) The genetic basis of inbreeding depression. Genetical Research, 74, 329-340.

Charlesworth B, Hughes KA (2000) The maintenance of genetic variation in life-history traits. In: Evolutionary Genetics: from Molecules to Morphology (eds Singh RS, Krimbas CB), pp. 369390. Cambridge University Press, Cambridge, UK. 
Charpentier M, Setchell JM, Prugnolle F et al. (2006) Life history correlates of inbreeding depression in mandrills (Mandrillus sphinx). Molecular Ecology, 15, 21-28.

Coltman D, Slate J (2003) Microsatellite measures of inbreeding: a meta-analysis. Evolution, 57, 971-983.

Coltman D, Pilkington J, Smith J, Pemberton J (1999) Parasitemediated selection against inbred Soay sheep in a free-living island population. Evolution, 53, 1259-1267.

Crnokrak P, Roff DA (1995) Dominance variance: associations with selection and fitness. Heredity, 75, 530-540.

Crnokrak P, Roff DA (1999) Inbreeding depression in the wild. Heredity, 83, 260-270.

Crow JF, Kimura M (1970) An Introduction to Population Genetics Theory. Harper \& Row, New York.

Curik I, Zechner P, Solkner J et al. (2003) Inbreeding, microsatellite heterozygosity, and morphological traits in Lipizzan horses. Journal of Heredity, 94, 125-132.

Daniels SJ, Walters JR (2000) Inbreeding depression and its effects on natal dispersal in red-cockaded woodpeckers. Condor, 102, 482-491.

Darwin C (1876) The Effects of Crossing and Self-fertilization in the Vegetable Kingdom. John Murray, London, UK.

David P (1998) Heterozygosity-fitness correlations: new perspectives on old problems. Heredity, 80, 531-537.

David P, Delay B, Berhou P et al. (1995) Alternative models for allozyme-associated heterosis in the marine bivalve Spisula ovalis. Genetics, 139, 1719-1726.

Dawson DA, Burke T, Hansson B et al. (2006) A predicted microsatellite map of the passerine genome based on chicken-passerine sequence similarity. Molecular Ecology, 15, 1299-1320.

De Rose MA, Roff DA (1999) A comparison of inbreeding depression in life history and morphological traits in animals. Evolution, 53, 1288-1292.

Double MC, Dawson D, Burke T, Cockburn A (1997) Finding the fathers in the least faithful bird: a microsatellite-based genotyping system for the superb fairy-wren Malurus cyaneus. Molecular Ecology, 6, 691-693.

Ellegren H (1999) Inbreeding and relatedness in Scandinavian grey wolves Canis lupus. Hereditas, 130, 239-244.

Engen S, Ringsby TH, Sather B-E et al. (in press) Effective size of fluctuating populations with two sexes and overlapping generations. Evolution.

Ferreira AGA, Amos W (2006) Inbreeding depression and multiple regions showing heterozygote advantage in Drosophila melanogaster exposed to stress. Molecular Ecology, 15, 3885-3893.

Frankham R (1998) Inbreeding and extinction: island populations. Conservation Biology, 12, 665-675.

Frankham R, Ballou JD, Briscoe DA (2002) Introduction to Conservation Genetics. Cambridge University Press, Cambridge, UK.

Garant D, Kruuk LEB (2005) How to use molecular marker data to measure evolutionary parameters in wild populations. Molecular Ecology, 14, 1843-1859.

Grant PR, Grant BR, Petren K (2001) A population founded by a single pair of individuals: establishment, expansion, and evolution. Genetica, 112-113, 359-382.

Griffith SC, Stewart IRK, Dawson DA, Owens IPF, Burke T (1999) Contrasting levels of extra-pair paternity in mainland and island populations of the house sparrow (Passer domesticus): is there an 'island effect'? Biological Journal of the Linnean Society, 68, 303-316.

Griffith SC, Owens IPF, Thuman KA (2002) Extra pair paternity in birds: a review of interspecific variation and adaptive function. Molecular Ecology, 11, 2195-2212.
Griffith SC, Dawson DA, Jensen H et al. (2007) Fourteen polymorphic microsatellite loci characterized in the house sparrows Passer domesticus (Passeridae, Aves). Molecular Ecology Notes, 7, 333-336.

Griffiths R, Double MC, Orr K, Dawson RJG (1998) A DNA test to sex most birds. Molecular Ecology, 7, 1071-1075.

Hanotte O, Zanon C, Pugh A et al. (1994) Isolation and characterization of microsatellite loci in a passerine bird: the reed bunting Emberiza schoeniclus. Molecular Ecology, 3, 529-530.

Hansson B, Westerberg L (2002) On the correlation between heterozygosity and fitness in natural populations. Molecular Ecology, 11, 2467-2474.

Hansson B, Bensch S, Hasselquist D, Åkesson M (2001) Microsatellite diversity predicts recruitment of sibling great reed warblers. Proceedings of the Royal Society of London. Series B, Biological Sciences, 268, 1287-1291.

Hansson B, Westerdahl H, Hasselquist D, Åkesson M, Bensch S (2004) Does linkage disequilibrium generate heterozygosity-fitness correlations in great reed warblers? Evolution, 58, 870-879.

Hansson B, Åkesson M, Slate J, Pemberton JM (2005) Linkage mapping reveals sex-dimorphic map distance in a passerine bird. Proceedings of the Royal Society of London. Series B, Biological Sciences, 272, 2289-2298.

Hartl DL, Clark AG (1997) Principles of Population Genetics, 3rd edn. Sinauer \& Associates, Sunderland, Massachusetts.

Hedrick P, Fredrickson R, Ellegren H (2001) Evaluation of d ${ }^{2}$, a microsatellite measure of inbreeding and outbreeding, in wolves with a known pedigree. Evolution, 55, 1256-1260.

Hedrick PW, Kalinowski ST (2000) Inbreeding depression in conservation biology. Annual Review of Ecology and Systematics, 31, 139-162.

Hughes C (1998) Integrating molecular techniques with field methods in studies of social behaviour: a revolution results. Ecology, 79, 383-399.

Husby A, Sæther B-E, Jensen H, Ringsby TH (2006) Causes and consequences of adaptive seasonal sex ratio variation in house sparrows. Journal of Animal Ecology, 75, 1128-1139.

Jeffery KJ, Keller LF, Arcese P, Bruford MW (2001) The development of microsatellite loci in the song sparrow, Melospiza melodia (Aves) and genotyping errors associated with good quality DNA. Molecular Ecology Notes, 1, 11-13.

Jensen H, Sæther B-E, Ringsby TH et al. (2003) Sexual variation in heritability and genetic correlations of morphological traits in house sparrow (Passer domesticus). Journal of Evolutionary Biology, 16, 1296-1307.

Jensen H, Sæther B-E, Ringsby TH et al. (2004) Lifetime reproductive success in relation to morphology in the house sparrow (Passer domesticus). Journal of Animal Ecology, 73, 599-611.

Jiménez JA, Hughes KA, Alaks G, Graham L, Lacy RC (1994) An experimental study of inbreeding depression in a natural habitat. Science, 266, 271-273.

Jones AG, Ardren WR (2003) Methods of parentage analysis in natural populations. Molecular Ecology, 12, 2511-2523.

Kashi YD, Soller M (1999) Functional roles of microsatellites and minisatellites. In: Microsatellites: Evolution and Applications (eds Schlötterer C Goldstein DB), pp. 10-23. Oxford University Press, Oxford, UK.

Keller LF (1998) Inbreeding and its fitness effects in an insular population of song sparrows (Melospiza melodia). Evolution, 52, 240-250.

Keller LF, Waller DM (2002) Inbreeding effects in wild populations. Trends in Ecology \& Evolution, 17, 230-241.

Keller LF, Arcese P, Smith JNM, Hochachka WM, Stearns SC 
(1994) Selection against inbred song sparrows during a natural bottleneck. Nature, 372, 356-357.

Keller LF, Grant PR, Grant BR, Petren K (2002) Environmental conditions affect the magnitude of inbreeding depression in survival of Darwin's finches. Evolution, 56, 1229-1239.

Kruuk LEB, Sheldon BC, Merila J (2002) Severe inbreeding depression in collared flycatchers (Ficedula albicollis). Proceedings of the Royal Society of London. Series B, Biological Sciences, 269, 1581-1589.

Lande R, Engen S, Sæther B-E (2003) Stochastic Population Dynamics in Ecology and Conservation. Oxford University Press, Oxford, UK.

Lynch M, Walsh B (1998) Genetics and Analyses of Quantitative Traits. Sinauer \& Associates, Sunderland, Massachusetts.

Malècot G (1969) The Mathematics of Heredity. Freeman, San Francisco, California.

Markert JA, Grant PR, Keller LF, Coombs JL, Petren K (2004) Neutral locus heterozygosity, inbreeding, and survival in Darwin's ground finches (Geospiza fortis and G. scandens). Heredity, 92, 306-315.

Marr AB, Arcese P, Hochachka WM, Reid JM, Keller LF (2006) Interactive effects of environmental stress and inbreeding on reproductive traits in a wild bird population. Journal of Animal Ecology, 75, 1406-1415.

Marshall TC, Slate J, Kruuk LEB, Pemberton JM (1998) Statistical confidence for likelihood-based paternity inference in natural populations. Molecular Ecology, 7, 639-655.

McArthur RH, Wilson EO (2001) The Theory of Island Biogeography. Princeton University Press, Princeton, New Jersey.

McRae SB (1996) Family values: costs and benefits of communal nesting in the moorhen. Animal Behaviour, 52, 225-245.

Menard S (2000) Coefficients of determination for multiple logistic regression analyses. American Statistician, 54, 17-24.

Neumann K, Wetton JH (1996) Highly polymorphic microsatellites in the house sparrow Passer domesticus. Molecular Ecology, 5, 307-309.

Overall ADJ, Byrne KA, Pilkington JG, Pemberton JM (2005) Heterozygosity, inbreeding and neonatal traits in Soay sheep on St Kilda. Molecular Ecology, 14, 3383-3393.

Pemberton J (2004) Measuring inbreeding depression in the wild: the old ways are the best. Trends in Ecology \& Evolution, 19, 613-615.

Pray LA, Schwartz JM, Goodnight CJ, Stevens L (1994) Environmental dependency of inbreeding depression: implications for conservation biology. Conservation Biology, 8, 562-568.

Ralls K, Ballou JD, Templeton A (1988) Estimates of lethal equivalents and the cost of inbreeding in mammals. Conservation Biology, 2, 185-193.

R Development Core Team (2004) R: A Language And Environment For Statistical Computing. R Development Core Team , Vienna, Austria. Available from URL: http://www.r-project.org.

Ringsby TH, Sæther B-E, Solberg EJ (1998) Factors affecting juvenile survival in house sparrow Passer domesticus. Journal of Avian Biology, 29, 241-247.

Ringsby TH, Sæther B-E, Solberg EJ (1999) Temporal and spatial variation in survival rates of a house sparrow, Passer domesticus, metapopulation. Oikos, 85, 419-425.

Ringsby TH, Sæther B-E, Tufto J, Jensen H, Solberg EJ (2002) Asynchronous spatiotemporal demography of a house sparrow metapopulation in a correlated environment. Ecology, 83, 561-569.

Roff DA (1998) Effects of inbreeding on morphological and life history traits in the sand cricket, Gryllus firmus. Heredity, 81, 28-37.

Saccheri I, Kuussaari M, Kankare M et al. (1998) Inbreeding and extinction in a butterfly metapopulation. Nature, 392, 491-494.

Sæther B-E, Ringsby TH, Bakke Ø, Solberg EJ (1999) Spatial and temporal variation in demography of a house sparrow metapopulation. Journal of Animal Ecology, 68, 628-637.
Savolainen O, Hedrick P (1995) Heterozygosity and fitness: no association in Scots pine. Genetics, 140, 755-766.

Sefc KM, Payne RB, Sorenson D (2001) Characterization of microsatellite loci in village indigobirds Vidua chalybeate and crossspecies amplification in estrildid and ploceid finches. Molecular Ecology Notes, 1, 252-254.

Slate J, Pemberton J (2002) Comparing moleculare measures for detecting inbreeding depression. Journal of Evolutionary Biology, 15, 20-31.

Slate J, Kruuk LEB, Marshall TC, Pemberton JM, Clutton-Brock TH (2000) Inbreeding depression influences lifetime breeding success in a wild population of red deer (Cervus elaphus). Proceedings of the Royal Society of London. Series B, Biological Sciences, 267, 1657-1662.

Slate J, Van Stijn TC, Anderson RM et al. (2002) A deer (subfamily Cervidae) genetic linkage map and the evolution of ruminant genomes. Genetics, 160, 1587-1597.

Slate J, David P, Dodds KG et al. (2004) Understanding the relationship between the inbreeding coefficient and multilocus heterozygosity: theoretical expectations and empirical data. Heredity, 93, 255-265.

Smith JNM, Keller LF, Marr AB, Arcese P (2006) Conservation and Biology of Small Populations. The Song Sparrows on Mandarte Island. Oxford University Press, New York.

Soulé ME (1986) Conservation Biology: The Science of Scarcity and Diversity. Sinauer \& Associates, Sunderland, Massachusetts.

Summers-Smith JD (1988) The Sparrows: A Study of the Genus Passer. $\mathrm{T} \&$ AD Poyser, Calton, UK.

Tufto J, Ringsby TH, Dhondt AA, Adriaensen F, Matthysen E (2005) A parametric model for estimation of dispersal patterns applied to five passerine spatially structured populations. American Naturalist, 165, E13-E26.

Van Noordwijk AJ, Scharloo W (1981) Inbreeding in an island population of the great tit. Evolution, 35, 674-688.

Walsh PS, Metzger DA, Higuchi R (1991) Chelex 100 as a medium for simple extraction of DNA for PCR-based typing from forensic material. BioTechniques, 10, 506-513.

Willi Y, Van Buskirk J, Hoffmann AA (2006) Limits to the adaptive potential of small populations. Annual Review of Ecology, Evolution and Systematics, 37, 433-458.

Wright S (1921) Systems of mating. Genetics, 6, 111-178.

Wright S (1969) Evolution and the Genetics of Populations: The Theory of Gene Frequencies, Vol. 2. University of Chicago Press, Chicago.

Wright S (1977) Evolution and the Genetics of Populations: Experimental Results and Evolutionary Deductions, Vol. 3. University of Chicago Press, Chicago.

Henrik Jensen is a research scientist employed at the Centre for Conservation Biology, NTNU. His research focuses mainly on problems in evolutionary and conservation biology, which he addresses using molecular methods and a long term house sparrow metapopulation study system. Erlend Myre Bremset is a former MSc-student whose MSc-project was part of the present study. He is now working as a high school teacher. Thor Harald Ringsby is a research scientist mainly working on questions in the field of population ecology and conservation biology. Bernt-Erik Sæther heads the Centre for Conservation Biology which focuses on research into dynamic changes in biological diversity at different organismic levels (genes, populations and communities) based on a stochastic modelling framework. 